\title{
Comparison of treatment delay associated with tunneled hemodialysis catheter placement between interventionists
}

Yoo Hyung Kim, Hae Ri Kim, Hong Jae Jeon, Ye-Jin Kim, Sa Ra Jung, Dae Eun Choi, Kang Wook Lee, and Ki Ryang $\mathrm{Na}$

Division of Nephrology, Department of Internal Medicine, Chungnam National University Hospital, Daejeon, Korea
Received: December 10, 2014

Revised : January 2, 2015

Accepted: January 9, 2015

\section{Correspondence to}

\section{Ki Ryang Na, M.D.}

Department of Internal Medicine, Chungnam National University

Hospital, 282 Munhwa-ro, Jung-

gu, Daejeon 35015, Korea

Tel: +82-42-280-7142

Fax: +82-42-280-7995

E-mail: drngr@cnu.ac.kr
Background/Aims: Fragmented care in nephrology can cause treatment delays. Nephrologists are qualified to perform vascular access-related procedures because they understand the pathophysiology of renal disease and perform physical examination for vascular access. We compared treatment delays associated with tunneled hemodialysis catheter (TDC) placement between interventional radiologists and nephrologists.

Methods: We collected data by radiologists from January 1, 2011 through December 31, 2011 and by nephrologists from since July 1, 2012 through June 30, 2013. We compared the duration from the hemodialysis decision to TDC placement (D-P duration) and hemodialysis initiation (D-H duration), catheter success and the complication rate, and the frequency and the usage time of non-tunneled hemodialysis catheters (NDCs) before TDC placement.

Results: The study analyzed 483 placed TDCs: 280 TDCs placed by radiologists and 203 by nephrologists. The D-P durations were 319 minutes (interquartile range [IQR], 180 to 1,057 ) in the radiologist group and 140 minutes (IQR, o to 792) in the nephrologist group. Additionally, the D-H durations were 415 minutes (IQR,260 to 1,091) and 275 minutes (IQR, 123 to 598), respectively. These differences were statistically significant $(p=0.00)$. The TDC success rate $(95.3 \%$ vs. $94.5 \%$, respectively; $p=0.32)$ and complication rate $(16.2 \%$ vs. $11 \%$, respectively; $p=0.11)$ did not differ between the groups. The frequency (24.5 vs. $26 \%$, respectively; $p=$ $0.72)$ and the usage time of NDC (8,451 vs. 8,416 minutes, respectively; $p=0.91)$ before TDC placement were not statistically significant.

Conclusions: Trained interventional nephrologists could perform TDC placement safely, minimizing treatment delays.

Keywords: Vascular access devices; Renal dialysis; Nephrology; Radiology

\section{INTRODUCTION}

Historically, there has been fragmented care in nephrology. Nephrologists have developed many tools to diagnose and manage kidney diseases. In addition, surgeons and interventional radiologists have played roles in vascular access management $[1,2]$. These forms of fragmented care of dialysis patients have led to delays in decision-making and treatment for patients [3]. To improve the prognosis of dialysis patients, since the 1990s, 
nephrologists in the United States have shown interest in vascular access management [4] and have recognized it as coordinated patient care, not an episodic event [5].

Nephrologists are ideally suited for vascular access-related procedures, including venography, percutaneous transvenous angioplasty and, in particular, tunneled hemodialysis catheter (TDC) placement because of their understanding of the pathophysiology of renal disease and mechanism of renal replacement therapy. These professionals can also perform physical examination for vascular access. In addition, they can determine the appropriate time for performance of a vascular procedure or initiation of hemodialysis. These characteristics might enable minimizing treatment delays and facilitate decision making for dialysis patients.

At our hospital, the responsibility for TDC placement was moved from the interventional radiologist to the interventional nephrologist. Therefore, a comparison of the treatment delay and safety and efficacy of TDC placement performed by interventional radiologists and interventional nephrologists is warranted.

\section{METHODS}

\section{Study design}

At Chungnam National University Hospital, interventional radiologists performed TDC placement until February 2012. Thereafter, interventional nephrologists performed vascular-access-related procedures, including TDC placement. In addition, non-tunneled hemodialysis catheter (NDC) placement has been performed by chief residents in the nephrology department.

To compare the outcomes of TDC placement, patients who underwent TDC placement by interventional radiologists for hemodialysis from January 1, 2011 to December 31, 2011 and by interventional nephrologists for hemodialysis from July 1, 2012 to June 30, 2013 were included in the present retrospective study. The medical records and radiological records were reviewed to examine patients' baseline characteristics, including age, sex, body mass index (BMI), comorbidities, reasons for TDC placement, information about hemodialysis, durations from the hemodialysis decision to TDC placement and hemodialysis initiation, and the complication rate.

We analyzed the duration from the hemodialysis deci- sion to TDC placement (D-P duration) and hemodialysis initiation (D-H duration) and the usage time of NDC as primary end points. To evaluate safety and efficacy, the frequency of NDC use, complication rate and success rate were analyzed as secondary end points.

\section{Interventionists}

In the interventional radiology department, there were two interventionists and one interventional X-ray machine. One interventionist had 11 years of experience and the other had 3 years of experience in a number of interventions including catheter insertion, arterial embolization, biopsy, and percutaneous transvenous angioplasty. In the interventional nephrology department, there was one interventionist who had observed the TDC placement procedure for 3 months and began performing the procedure in March 2012. We began to collect data for the interventional nephrologist after placement of 18 TDCs on his own.

Both departments used the same method with a different type of catheter, either the step-tip catheter or palindrome catheter. The internal jugular vein was punctured with a $20-\mathrm{G}$ needle under sonographic guidance. The guide-wire was placed via the needle, which was then removed. Thereafter, the anterior chest was anesthetized with lidocaine. A tunneler was used to create a tunnel from the skin of the upper anterior chest to the internal jugular vein puncture site. Either a palindrome catheter (for the interventional nephrologist) or a step-tip catheter (for the interventional radiologist) were inserted into the tunnel. Staged dilation was performed via the guide-wire using 12-French (Fr), 14-Fr, and 16-Fr catheters, followed by 'valved pull apart sheath' insertion. Inside the valved pull apart sheath, the TDC was inserted and the valved pull apart sheath was pulled apart. The internal jugular vein puncture site and upper anterior chest puncture site were then sutured closed.

\section{Definitions and classifications}

The reasons for TDC placement were divided into six categories: (1) acute kidney injury (AKI); (2) end-stage renal disease (ESRD); (3) change in hemodialysis modality from peritoneal dialysis to hemodialysis; (4) arteriovenous fistula (AVF) or graft (AVG) malfunction; (5) for continuous renal replacement therapy or plasmapheresis; and (6) TDC reinsertion due to TDC malfunction 
or infection.

The consultation time was assumed to be the 'hemodialysis decision time' in both groups. The TDC placement time was identified in the procedure sheet through medical chart review. The hemodialysis initiation time was taken to be the time recorded on the hemodialysis sheet. The D-P duration was defined as the number of minutes from the consultation time to the TDC placement time, and the $\mathrm{D}-\mathrm{H}$ duration was defined as the number of minutes from the consultation time to the hemodialysis initiation time.

For the patients who used NDC, the usage time of NDC was defined as the number of minutes from the initial hemodialysis time to the TDC placement time.

Regarding complications, cardiothoracic complications were defined as cardiothoracic problems related to the procedure within 1 month of TDC placement. Minor bleeding was defined as delayed bleeding within five hemodialysis sessions after TDC placement that required no additional therapy and that resolved spontaneously. Major bleeding was defined as delayed bleeding within five hemodialysis sessions after TDC placement that required additional therapy and that resulted in hospitalization.

Catheter success was defined no disturbance of blood flow within five hemodialysis sessions immediately after TDC placement was completed. A failed catheter placement was defined as catheter placement that did not meet the criteria for success. An aborted catheter placement was defined as catheter placement that was attempted but could not be completed.

\section{Statistical analyses}

Continuous variables-including age, BMI, and delay time-were expressed as means \pm standard deviation, and statistical significance was compared by Student $t$ test if the $p$ value in the Kolmogorov-Smirnov test was greater than 0.05 . If not, the median value was used to evaluate statistical significance with the Mann-Whitney $U$ test. Categorical data, including the catheter type, catheter placement side, comorbidities, reasons for TDC placement and complication rate were analyzed by crosstab. The SPSS version 18.0 (SPSS Inc., Chicago, IL, USA) was used. The null hypothesis of no difference was rejected if the $p$ value was lower than 0.05 or equivalent.

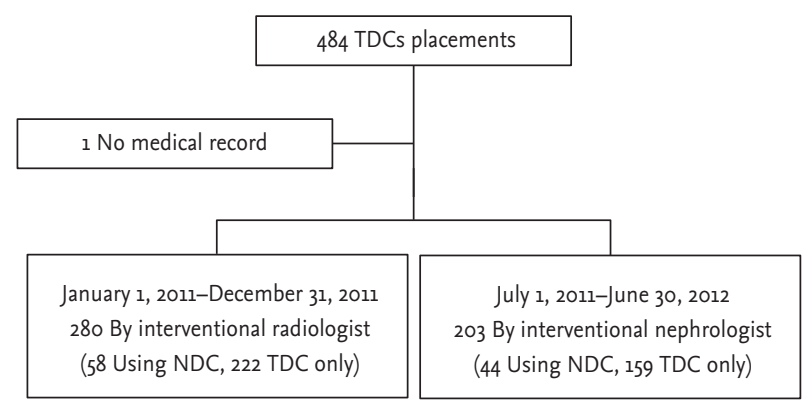

Figure 1. Study design. TDC, tunneled hemodialysis catheter; NDC, non-tunneled hemodialysis catheter.

\section{RESULTS}

\section{Baseline characteristics}

We assessed 484 TDCs by interventional radiologists and interventional nephrologists. Among them, one case was excluded because no medical record was found. Finally, 280 cases by radiologists and 203 cases by nephrologists were included (Fig. 1).

The baseline characteristics, including age, sex, and BMI, were not significantly different between the two groups. Comorbidities showed a similar distribution between the two groups, but patients in the nephrologist group had a higher frequency of diabetes (radiologists vs. nephrologists: $42.1 \%$ vs. $53.2 \%$, respectively; $p=$ 0.02). Reasons for TDC placement also showed a similar distribution, but TDC placement occurred due to ESRD more frequently in the nephrologist group than in the radiologist group ( $49.6 \%$ vs. $60.6 \%, p=0.02$ ).

There were large differences between the two groups in the catheters used. The radiologists used the step-tip catheter with the exception of one case, and the nephrologists used a palindrome catheter in all but five cases (99.6\% vs. $2.5 \%$ for the step-tip catheter; $0.4 \%$ vs. $97.5 \%$ for the palindrome catheter, $p=0.00$ in both groups). In addition, the right internal jugular vein was the favored side in both groups, but a higher proportion of TDCs was placed in the right internal jugular vein in the nephrologist group (87.8\% vs. 93.5\%; $p=0.04$ ) (Table 1 ).

\section{Time from the hemodialysis decision to TDC place- ment (D-P duration)}

In the radiologist and nephrologist groups, the overall durations from the hemodialysis decision to TDC placement were 319 minutes (interquartile range [IQR], 
Table 1. Baseline characteristics

\begin{tabular}{|c|c|c|c|}
\hline Characteristic & By radiologist $(n=280)$ & By nephrologist $(n=203)$ & $p$ value \\
\hline Age, yr & $63.77 \pm 14.82$ & $64.94 \pm 13.24$ & 0.37 \\
\hline Female sex & $107(38.2)$ & $92(45 \cdot 3)$ & 0.12 \\
\hline Body mass index, $\mathrm{kg} / \mathrm{m}^{2}$ & $22.6 \pm 3.79$ & $23.0 \pm 4.66$ & 0.38 \\
\hline \multicolumn{4}{|l|}{ Catheter } \\
\hline Palindrome & $1(0.4)$ & $198(97.5)$ & 0.00 \\
\hline Step-tip catheter & $279(99.6)$ & $5(2.5)$ & 0.00 \\
\hline Right:Left & $246(87.8): 34(12.1)$ & $190(93.5): 13(6.4)$ & 0.04 \\
\hline \multicolumn{4}{|l|}{ Comorbidities } \\
\hline Diabetes mellitus & $118(42.1)$ & $108(53.2)$ & 0.02 \\
\hline Hypertension & $120(42.9)$ & $91(44.8)$ & 0.66 \\
\hline Heart failure & $41(14.6)$ & $36(17.7)$ & 0.36 \\
\hline Cerebral infarction & $37(13.2)$ & $26(12.8)$ & 0.89 \\
\hline Glomerulonephritis & $28(10.0)$ & $22(10.8)$ & 0.76 \\
\hline Liver cirrhosis & $19(6.8)$ & $12(5 \cdot 9)$ & 0.69 \\
\hline COPD & $11(3 \cdot 9)$ & $2(1.0)$ & 0.05 \\
\hline PCKD & $6(2.1)$ & 0 & 0.04 \\
\hline \multicolumn{4}{|l|}{ Reason for dialysis } \\
\hline ESRD & $139(49.6)$ & $123(60.6)$ & 0.02 \\
\hline Acute kidney injury & $50(17.9)$ & $28(13.8)$ & 0.23 \\
\hline From PD to HD & $10(3.6)$ & $5(2.5)$ & 0.48 \\
\hline Catheter reinsertion & $42(15)$ & $31(15 \cdot 3)$ & 0.93 \\
\hline AVF or AVG failure & $38(13.6)$ & $16(7 \cdot 9)$ & 0.05 \\
\hline CRRT & $1(0.4)$ & 0 & 1 \\
\hline
\end{tabular}

Values are presented as mean $\pm \mathrm{SD}$ or number (\%).

COPD, chronic obstructive pulmonary disease; PCKD, polycystic kidney disease; ESRD, end-stage renal disease; PD, peritoneal dialysis; HD, hemodialysis; AVF, arteriovenous fistula; AVG, arteriovenous graft; CRRT, continuous renal replacement therapy.

180 to 1,057$)$ and 140 minutes (IQR, o to 792), respectively; this difference was statistically significant $(p=0.00)$.

In cases using NDC before TDC placement, the D-P durations in both groups were increased significantly $(p=0.01)$ to 1,473 minutes (IQR, 581 to 2,898$)$ and 640 minutes (IQR, 69 to 1,545); however, in cases without the use of NDC before TDC placement, the D-P duration in both groups were decreased $(p=0.00)$ to 259 minutes (IQR, 157 to 499) and 97 minutes (IQR, o to 315) in the radiologist and nephrologist groups, respectively (Fig. 2).

\section{Time from hemodialysis decision to hemodialysis initiation ( $D-H$ duration)}

Before comparison of the D-H duration, we compared the delay between TDC placement and hemodialysis initiation in the two groups. The times between TDC placement to hemodialysis initiation were 83 minutes (IQR, 54 to 132) in the radiologist group and 103 minutes (IQR, 71 to 150$)$ in the nephrologist group $(p=0.00)$. The time from TDC placement to hemodialysis initiation was delayed by a further 20 minutes in the nephrologist group.

Importantly, the $\mathrm{D}-\mathrm{H}$ duration also showed statistically significant differences. Overall, the D-H durations in the radiologist and nephrologist groups were 415 minutes (IQR, 260 to 1,091) and 275 minutes (IQR, 123 to 598), respectively; the difference was statistically significant $(p=0.00)$. In cases using NDC before TDC placement, the $\mathrm{D}-\mathrm{H}$ durations in both groups were significantly increased $(p=0.00)$ to 1,593 minutes (IQR, 950 to 3,000) and 617 minutes (IQR, 231 to 1,700), respectively. In cases without NDC before TDC placement, the D-H 
Table 2. Complications and success rates

\begin{tabular}{lccc}
\hline Variable & By radiologist $(\mathrm{n}=278)$ & By nephrologist $(\mathrm{n}=200)$ & $p$ value \\
\hline Minor bleeding & $45(16.2)$ & $22(11.0)$ & 0 \\
Major bleeding & $1(0.4)$ & 0.11 & 0.58 \\
Success rate & $265(95.3)$ & $189(94.5)$ & 0.32 \\
\hline
\end{tabular}

Values are presented as number (\%).



Figure 2. Time from the hemodialysis decision to TDC placement (D-P duration) in each group. In the radiologist group, D-P duration was longer compared to the nephrologist group in each comparison; overall, TDC alone and $\mathrm{NDC} \rightarrow$ TDC. TDC, tunneled hemodialysis catheter; NDC, non-tunneled hemodialysis catheter. ${ }^{\mathrm{a}} \mathrm{p}=0.00$ vs. radiologist, ${ }^{b} p=0.01$ vs. radiologist.

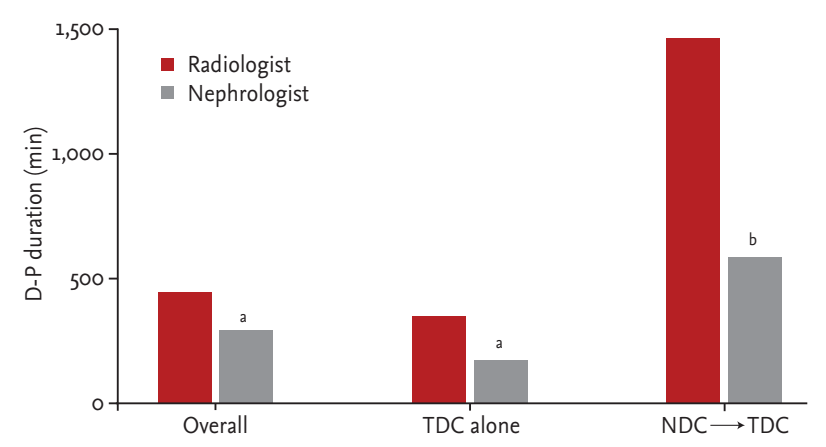

Figure 3. Time from the hemodialysis decision to hemodialysis initiation (D-H duration). In the radiologist group, D-H duration was longer compared to the nephrologist group in each comparison; overall, TDC alone and NDC $\rightarrow$ TDC. TDC, tunneled hemodialysis catheter; NDC, non-tunneled hemodialysis catheter. ${ }^{a} p=0.00$ vs. radiologist, ${ }^{b} p=0.01$ vs. radiologist.

durations in both groups were decreased $(p=0.01)$ to 360 minutes (IQR, 240 to 616 ) and 223 minutes (IQR, 119 to 422), respectively (Fig. 3).


Figure 4. Frequency of non-tunneled hemodialysis catheter (NDC) use. A, overall comparison of NDC frequency $(p=0.72)$; $\mathrm{B}$, tunneled hemodialysis catheter (TDC) placement due to acute kidney injury (AKI) or end-stage renal disease (ESRD) excluding intensive care unit (ICU) cases $(p=0.74)$; C, TDC placement due to ESRD excluding ICU cases $(p=0.34)$.

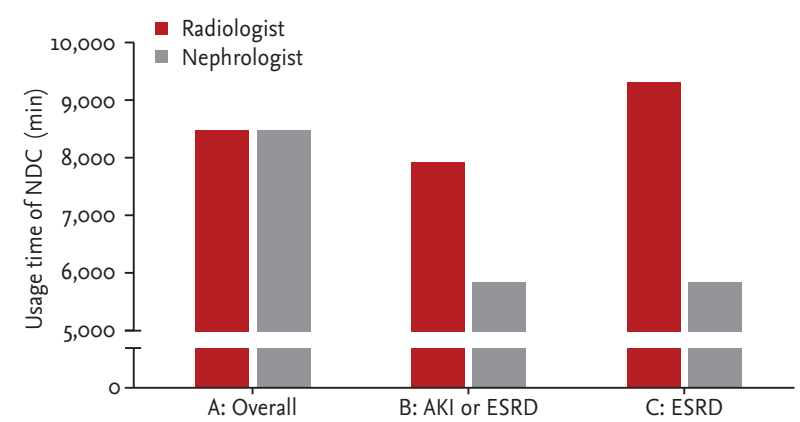

Figure 5. The usage time of non-tunneled hemodialysis catheter (NDC) before tunneled hemodialysis catheter (TDC) placement. A, overall comparison of the usage time of NDC $(p=0.91) ; \mathrm{B}, \mathrm{TDC}$ placement due to acute kidney injury (AKI) or end-stage renal disease (ESRD) excluding intensive care unit (ICU) cases $(p=0.49)$; C, TDC placement due to ESRD excluding ICU cases $(p=0.14)$.

\section{Frequency and usage time of NDC}

The frequency of NDC use was analyzed. After excluding duplicated patients, 58 of 237 patients (24.5\%) in the radiologist group and 44 of 169 patients (26\%) in the 
nephrologist group used NDC before TDC placement; this difference was not significant $(p=0.72)$ (Fig. 4). We performed subgroup analysis to match disease severity and reasons for TDC placement in the two groups. Only patients who underwent TDC placement due to AKI and ESRD and who were not in the intensive care unit (ICU) were analyzed additionally. Forty-one patients (26.1\%) used NDC in the radiologist group, and $29(24.4 \%)$ in the nephrologist group; this difference was not significant $(p=0.74)$. Only cases with ESRD and non-ICU cases were analyzed. Thirty-two patients (25.8\%) used NDC before TDC placement in the radiologist group, compared to 21 patients $(20.4 \%)$ in the nephrologist group. Thus a lower percentage of patients used NDC before TDC placement in the nephrologist group, but the difference was not significant $(p=0.34)$.

We compared the usage time of NDC because we already reported reduced D-P duration and D-H duration in the nephrology group, particularly in cases using NDC. There were 58 patients in the radiologist group and 44 in the nephrology group. Overall, the usage times for the NDC were 8,451 minutes (IQR, 4,141 to 12,759) in the radiologist group and 8,416 minutes (IQR, 3,982 to $12,458)$ in the nephrologist group. This difference was not statistically significant ( $p=0.91$ ) (Fig. 5).

To match the patients' disease severity and reasons for TDC placement between the two groups, we excluded ICU cases and included cases of only AKI or ESRD. In subgroup analysis, the usage time of NDC was 7,899 minutes (IQR, 3,982 to 12,685) in the radiologist group and 5,867 minutes (IQR, 3,953 to 9,884) in the nephrologist group; this difference was not statistically significant $(p=0.49)$ (Fig. 5). In a subgroup analysis of only ESRD patients, the usage time of NDC was 9,300 minutes (IQR, 5,134 to 13,752) and 5,867 minutes (IQR, 3,953 to $9,884)(p=0.14)$, respectively (Fig. 5). This analysis showed a trend towards greater differences in duration between the two groups in the process of subgrouping.

\section{Complication rates}

Bleeding was the only complication in both groups. Minor bleeding occurred at a frequency of $16.2 \%$ in the radiologist group and $11 \%$ in the nephrologist group. We found a trend towards a decreasing complication rate in the nephrologist group. However, there was no statistical significance $(p=0.11)$ (Table 2).

\section{Success rates}

The success rates were $95.3 \%$ in the radiologist group and $94.5 \%$ in the nephrologist group. This difference was not statistically significant $(p=0.32)$ (Table 2).

\section{DISCUSSION}

In the mid-1980s, a silicone dual lumen with a Dacron-cuffed hemodialysis catheter in the internal jugular vein became an alternative vascular access to a prosthetic graft or subclavian catheter [6-8], and its use increased thereafter. In 2003, to reduce the morbidity and mortality associated with vascular access and medical budget, the Fistula First Initiative (FFI) was implemented [9]. Although a greater number of ESRD patients had fistulas created before hemodialysis initiation after FFI, the critical challenge was achieving functioning fistulas. As the use of AVF increased, the more frequent was the use of TDC $[10,11]$ as a bridge until AVF maturation.

Moreover, the percentage of patients who used TDC as a primary vascular access has increased and an 'increasing TDC use, reducing graft use and stable AVF' trend has been reported in many countries $[12,13]$.

In addition to TDC placement, TDC maintenance and management and changing vascular access from TDC to AVF are also important issues because they are closely associated with patient mortality and morbidity [13-16]. In this context, vascular access care has been recognized as coordinated patient care, not an episodic event [5]. Of course, nephrologists are suited for this task.

Many obstacles can prevent nephrologists from starting interventions, which are associated with vascular access. The cause is mainly a lack of experience in the use of the procedural tools, conflict between radiologists and surgeons who may have already participated in vascular access-related procedures, lack of an education program and fear of acute or delayed complications and their management, including hemopneumothorax, arrhythmia, vessel rupture, bleeding, catheter malfunction, recirculation, procedure failure and infection [17].

However, Beathard et al. [18] showed that trained interventional nephrologists at multiple centers could perform TDC placements effectively and safely. Asif et al. [5] showed that interventional nephrologists minimized treatment delays, decreased hospitalization duration and 
temporary catheter use and improved patient comfort. It would be interesting to determine the amount of time that could be saved if nephrologists participated in vascular access-related procedures.

In this study, the D-P duration was reduced by nephrologists by 179 minutes (2.9 hours) compared to radiologists. For patients who used an NDC before TDC placement, the difference in the D-P duration between the two groups was increased by 833 minutes (13.8 hours). Treatment delay was reduced by 2.9 hours in the overall comparison and by 13.8 hours if patients used NDC, compared to equivalent times by radiologists. In addition, the differences of the D-P durations in each subgroup with or without NDC before TDC placement were 1,214 minutes in the radiologist group and 543 minutes in the nephrologist group, which suggests that nephrologists attempted to minimize usage time of NDC and changed the vascular access from NDC to TDC earlier for patients in whom hemodialysis had to be maintained.

The frequency of NDC use was not different between the two groups, as expected. Many variables likely determined whether patients required NDC before TDC placement for hemodialysis, including emergency, disease severity, and night-time and weekend placement. However, after adjusting disease severity through excluding ICU cases and reasons for TDC placement, the frequency of NDC use was stable in the radiologist group and decreased in the nephrologist group, although the change was not statistically significant. Among patients who required permanent hemodialysis, the interventional nephrologist attempted to place more TDCs and fewer NDCs than the interventional radiologist because TDC placement was one of hemodialysis patient care, not just one of interventions to interventional nephrologist.

We expected that the usage time of NDC would be reduced if nephrologists performed TDC placement because they would reduce the D-P and D-H durations compared to radiologists. In a subgroup analysis of only ESRD patients, the usage time of NDC was reduced in the nephrologist group by 3,433 minutes (57 hours), but the difference was not statistically significant. In the subgroup analysis, the numbers of patients were reduced to 32 and 21, respectively; however, the standard deviation was too large for statistical significance to be detected.

We compared the number of NDC exchanged due to an adverse event before TDC placement. Excluding ICU cases, three NDCs $(6.3 \%)$ were exchanged due to catheter related infection among 47 NDCs in the radiologist group and there was no case of NDC exchange in the nephrologist group. Additionally, five NDCs (10.6\%) in the radiologist group and three NDCs (10.3\%) among 29 NDCs in the nephrologist group were exchanged due to a malfunctioning catheter. As forementioned, the 3,433-minute (57 hours) usage time of the NDC was too short to allow catheter-related problems to occur. In order to assign statistical and clinical significance to data of catheter related infections, we required more variables to adjust, including the type of procedure, type of catheter, and level of aseptic technique.

Various factors are related to the function and survival of TDC. Kidney Disease Outcomes Quality Initiative (K-DOQI) recommends that TDC be placed in the right internal jugular vein. Catheter placement on the other side causes a greater number of complications [19-22]. Poorer blood flow rates and greater percentages of stenosis and thrombosis are associated with hemodialysis catheter placement in the left jugular vein $[6,21]$. The type of catheter used also influences catheter outcomes. Use of a palindrome catheter with a spiral-z tip design compared to other types of catheters is associated with lower thrombosis and reintervention rates [23,24]. In addition, a palindrome catheter was reported to reduce recirculation [24,25]. In our study, nephrologists attempted to place TDC in the right jugular vein and used palindrome catheters in most cases. This suggested that patients could benefit from the contributions of interventional nephrologists who considered TDC placement not as an episodic procedure, but as coordinated patient care.

The success and complication rates were compared to analyze the effectiveness and safety of TDC placement. In both groups, acute complications involved only bleeding, not vessel rupture or hemopneumothorax. Although one major bleeding and a higher rate of minor bleeding occurred in the radiologist group, the difference was not statistically significant. The success rates were similar in the two groups. Therefore, nephrologists could perform TDC placement effectively and safely.

The main limitation of our study was its retrospective 
observational nature; such studies are subject to many sources of confounding bias. First, in our study, most of the patients were lost to follow up; thus, we could not compare the long-term survival of TDC. In addition, we could not describe constructional educational programs for training of interventional nephrologists. Thirdly, if we could compare more NDC cases, we could report the effects of treatment delays including the frequency and usage time of NDC.

However, to compare the treatment delay between groups, a retrospective observational study design is needed because the interventionist's purpose to minimize delay must be excluded. Moreover, a large number of TDC cases were included in this study, and the baseline characteristics of the two groups were relatively similar.

In conclusion, interventional nephrologists could minimize the D-P duration and D-H duration. The success and complication rates were not significantly different from those of interventional radiologists, indicating that nephrologists could place TDC effectively and safely. Our results showed that TDC placement by nephrologists enhances patient comfort and minimizes hospitalization.

\section{KEY MESSAGE}

1. Interventional nephrologist could reduce the treatment delay regarding tunneled hemodialysis catheter placement.

2. The success and complication rate of the interventional nephrologist were similar that of the interventional radiologist.

\section{Conflict of interest}

No potential conflict of interest relevant to this article was reported.

\section{REFERENCES}

1. Lund GB, Trerotola SO, Scheel PF Jr, et al. Outcome of tunneled hemodialysis catheters placed by radiologists. Radiology 1996;198:467-472.

2. Trerotola SO, Johnson MS, Harris VJ, et al. Outcome of tunneled hemodialysis catheters placed via the right internal jugular vein by interventional radiologists. Radiology 1997;203:489-495.

3. Asif A, Byers P, Vieira CF, Preston RA, Roth D. Diagnostic and interventional nephrology. Am J Ther 2002;9:530-536.

4. Jackson JW, Lewis JL, Brouillette JR, Brantley RR Jr. Initial experience of a nephrologist-operated vascular access center. Semin Dial 2000;13:354-358.

5. Asif A, Besarab A, Roy-Chaudhury P, Spergel LM, Ravani P. Interventional nephrology: from episodic to coordinated vascular access care. J Nephrol 2007;20:399-405.

6. Moss AH, McLaughlin MM, Lempert KD, Holley JL. Use of a silicone catheter with a Dacron cuff for dialysis short-term vascular access. Am J Kidney Dis 1988;12:492498.

7. Moss AH, Vasilakis C, Holley JL, Foulks CJ, Pillai K, McDowell DE. Use of a silicone dual-lumen catheter with a Dacron cuff as a long-term vascular access for hemodialysis patients. Am J Kidney Dis 1990;16:211-215.

8. Schwab SJ, Buller GL, McCann RL, Bollinger RR, Stickel DL. Prospective evaluation of a Dacron cuffed hemodialysis catheter for prolonged use. Am J Kidney Dis 1988;11:166-169.

9. Lok CE. Fistula first initiative: advantages and pitfalls. Clin J Am Soc Nephrol 2007;2:1043-1053.

10. Ackad A, Simonian GT, Steel K, et al. A journey in reversing practice patterns: a multidisciplinary experience in implementing DOQI guidelines for vascular access. Nephrol Dial Transplant 2005;20:1450-1455.

11. Wasse H, Speckman RA, Frankenfield DL, Rocco MV, McClellan WM. Predictors of delayed transition from central venous catheter use to permanent vascular access among ESRD patients. Am J Kidney Dis 2007;49:276-283.

12. Moist LM, Trpeski L, Na Y, Lok CE. Increased hemodialysis catheter use in Canada and associated mortality risk: data from the Canadian Organ Replacement Registry 2001-2004. Clin J Am Soc Nephrol 2008;3:1726-1732.

13. Rayner HC, Besarab A, Brown WW, Disney A, Saito A, Pisoni RL. Vascular access results from the Dialysis Outcomes and Practice Patterns Study (DOPPS): performance against Kidney Disease Outcomes Quality Initiative (K/ DOQI) Clinical Practice Guidelines. Am J Kidney Dis 2004;44(5 Suppl 2):22-26.

14. Astor BC, Eustace JA, Powe NR, et al. Type of vascular access and survival among incident hemodialysis patients: the Choices for Healthy Outcomes in Caring for ESRD 
(CHOICE) Study. J Am Soc Nephrol 2005;16:1449-1455.

15. Dhingra RK, Young EW, Hulbert-Shearon TE, Leavey SF, Port FK. Type of vascular access and mortality in U.S. hemodialysis patients. Kidney Int 2001;60:1443-1451.

16. Polkinghorne KR, McDonald SP, Atkins RC, Kerr PG. Vascular access and all-cause mortality: a propensity score analysis. J Am Soc Nephrol 2004;15:477-486.

17. Vesely TM, Beathard G, Ash S, Hoggard J, Schon D; ASDIN Clinical Practice Committee. A position statement from the American Society of Diagnostic and Interventional Nephrology. Semin Dial 2007;20:359-364.

18. Beathard GA, Litchfield T; Physician Operators Forum of RMS Lifeline, Inc. Effectiveness and safety of dialysis vascular access procedures performed by interventional nephrologists. Kidney Int 2004;66:1622-1632.

19. Cimochowski GE, Worley E, Rutherford WE, Sartain J, Blondin J, Harter H. Superiority of the internal jugular over the subclavian access for temporary dialysis. Nephron 1990;54:154-161.

20. Schillinger F, Schillinger D, Montagnac R, Milcent T.
Post-catheterization venous stenosis in hemodialysis: comparative angiographic study of 50 subclavian and 50 internal jugular accesses. Nephrologie 1992;13:127-133.

21. Vanholder R, Ringoir S. Vascular access for hemodialysis. Artif Organs 1994;18:263-265.

22. Develter W, De Cubber A, Van Biesen W, Vanholder R, Lameire N. Survival and complications of indwelling venous catheters for permanent use in hemodialysis patients. Artif Organs 2005;29:399-405.

23. Kakkos SK, Haddad GK, Haddad RK, Scully MM. Effectiveness of a new tunneled catheter in preventing catheter malfunction: a comparative study. J Vasc Interv Radiol 2008;19:1018-1026.

24. Hwang HS, Kang SH, Choi SR, Sun IO, Park HS, Kim Y. Comparison of the palindrome vs. step-tip tunneled hemodialysis catheter: a prospective randomized trial. Semin Dial 2012;25:587-591.

25. Tal MG. Comparison of recirculation percentage of the palindrome catheter and standard hemodialysis catheters in a swine model. J Vasc Interv Radiol 2005;16:1237-1240. 